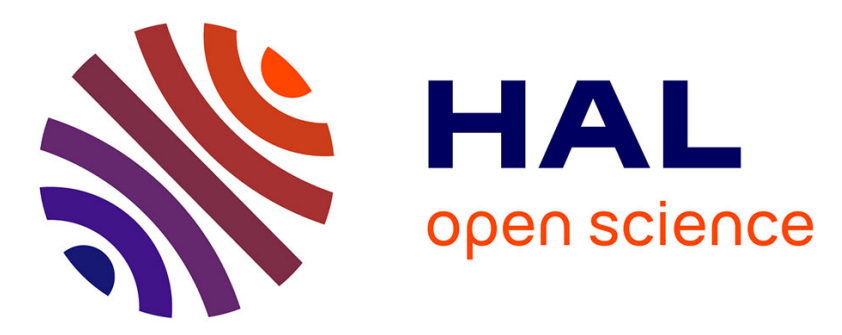

\title{
Robust and Optimal Registration of Image Sets and Structured Scenes via Sum-of-Squares Polynomials
}

Danda Pani Paudel, Adlane Habed, Cédric Demonceaux, Pascal Vasseur

\section{To cite this version:}

Danda Pani Paudel, Adlane Habed, Cédric Demonceaux, Pascal Vasseur. Robust and Optimal Registration of Image Sets and Structured Scenes via Sum-of-Squares Polynomials. International Journal of Computer Vision, 2019, 127 (5), pp.415-436. 10.1007/s11263-018-1114-2 . hal-02113657

\section{HAL Id: hal-02113657 \\ https://hal.science/hal-02113657}

Submitted on 23 Mar 2021

HAL is a multi-disciplinary open access archive for the deposit and dissemination of scientific research documents, whether they are published or not. The documents may come from teaching and research institutions in France or abroad, or from public or private research centers.
L'archive ouverte pluridisciplinaire $\mathbf{H A L}$, est destinée au dépôt et à la diffusion de documents scientifiques de niveau recherche, publiés ou non, émanant des établissements d'enseignement et de recherche français ou étrangers, des laboratoires publics ou privés. 


\title{
Robust and Optimal Registration of Image Sets and Structured Scenes via Sum-of-Squares Polynomials
}

\author{
Danda Pani Paudel • Adlane Habed • Cédric Demonceaux • Pascal Vasseur.
}

Received: date / Accepted: date

\begin{abstract}
This paper addresses the problem of registering a known structured 3D scene, typically a 3D scan, and its metric Structure-from-Motion (SfM) counterpart. The proposed registration method relies on a prior plane segmentation of the $3 \mathrm{D}$ scan. Alignment is carried out by solving either the point-to-plane assignment problem, should the SfM reconstruction be sparse, or the plane-to-plane one in case of dense SfM. A polynomial Sum-of-Squares optimization theory framework is employed for identifying point-to-plane and plane-toplane mismatches, i.e. outliers, with certainty. An inlier set maximization approach within a Branch-and-Bound search scheme is adopted to iteratively build potential inlier sets and converge to the solution satisfied by the largest number of assignments. Plane visibility conditions and vague camera locations may be incorporated for better efficiency without sacrificing optimality. The registration problem is solved in two cases: (i) putative correspondences (with possibly overwhelmingly many outliers) are provided as input and (ii) no initial correspondences are available. Our approach yields outstanding results in terms of robustness and optimality.
\end{abstract}

Keywords 2D-3D registration - Structure-fromMotion · Polynomial Sum-of-Squares optimization

\section{Paudel}

Computer Vision Lab, ETH Zurich, Switzerland E-mail: paudel@vision.ee.ethz.ch

A. Habed

ICube laboratory, University of Strasbourg, CNRS, France

E-mail: adlane.habed@icube.unistra.fr

C. Demonceaux

Le2i laboratory, University of Bourgogne Franche-Comté, CNRS, France E-mail: cedric.demonceaux@u-bourgogne.fr

P. Vasseur

LITIS EA laboratory, University of Rouen, France E-mail: pascal.vasseur@univ-rouen.fr

\section{Introduction}

The emergence of affordable 3D sensors and high quality $2 \mathrm{D}$ cameras has triggered a growing interest in combining both imaging modalities. 3D sensors provide faithful 3D scene models in the form of dense 3D point clouds while images are used to extract texture information. High quality 3D models with mapped texture can be obtained so long as the $2 \mathrm{D}$ and $3 \mathrm{D}$ sensors are registered in a common reference frame. The two modalities are generally registered off-line and the $2 \mathrm{D}$ and $3 \mathrm{D}$ sensors kept rigidly attached during acquisition. Doing so, however, may prove impractical considering that suitable acquisition conditions for one sensor may not be adequate for the other (e.g. lighting conditions for cameras, surface orientation for 3D sensor, etc.). Some applicationspecific requirements (e.g. camera on a drone and a $3 \mathrm{D}$ scanner on a vehicle) may altogether prohibit the sensors to be rigidly attached. When the $2 \mathrm{D}$ and $3 \mathrm{D}$ sensors are free, reliable methods for registering the two modalities, i.e. establishing inter-modality correspondences and estimating the rigid transformation aligning their reference frames, are highly desirable.

Structure-from-Motion (SfM) techniques compute $3 \mathrm{D}$ point coordinates from pixel correspondences across images. It is thus tempting to regard the problem of registering $3 \mathrm{D}$ and $2 \mathrm{D}$ sensors as that of aligning two $3 \mathrm{D}$ point sets: one set induced by the images and the other obtained from scanner measurements. Registering 3D point clouds is a well-studied problem. Most methods use the Iterative-Closest-Point (ICP) algorithm (or its variants) [33, 13, 27. While ICP is a local method, recent work by Yang et. al [36] (Go-ICP) provides the very first globally optimal solution to same-scale point set registration. However, because SfM reconstructions 
suffer from a scale ambiguity, methods devised for registering same-scale data cannot be employed.

Most methods handling the scale ambiguity rely on establishing correspondences either between the 3D measurements obtained by both modalities or directly between scanned data and images [19, 7, 10. The sought transformation parameters are then obtained by either minimizing the registration loss function or maximizing the consensus set of inliers. Note that Random Sample Consensus (RANSAC) [12] is the most widely used method for finding the set of maximum inliers. Methods based on loss function minimization are more prone to outliers than their inlier-set-maximization counterparts [1. Some approaches exploit scene knowledge or the Manhattan World assumption. For instance, methods have been devised based on line segment matching [19, target segmentation [32, repeated patterns detection 28, mutual information maximization [20, and extended Chamfer matching [38. Registration methods relying on establishing correspondences may be undermined by unreliable visual feature descriptors. Alternative methods, not establishing initial correspondences, have also been proposed [23, 9, 8, 24]. The methods in 223, 9] use variants of the ICP algorithm and hence remain susceptible to partial scene overlap, scene occlusion, and high levels of outliers. The one in $[8]$ employs a RANSAC-based inlier set maximization in which the scale problem is handled by an extension of the 4-point congruent sets algorithm. A recent approach presented in 24. computes the average gradient magnitude over all lighting directions under Lambertian shading. These gradients are then matched with the image gradient to obtain a coarse 2D-3D registration.

As far as maximizing the set of inliers is concerned, RANSAC is non-deterministic and provides no guarantee with respect to the optimality of its solution. Globally optimal inlier set maximization methods 18, 1] have recently been proposed for problems formulated through linear equations. Extensions to problems with nonlinear equations [37] is problem-specific, difficult and may result in much more complicated (possibly numerically intractable) mathematical formulations. Note that a variety of methods for solving systems of nonlinear polynomial equations exist. Some are based on Gröbner bases or homotopy continuation 34 , 14. Others use polynomial Sum-of-Squares (SoS) optimization 29, 17, 22, 21, 4, 5. However, such methods are dedicated to solving outlier-free systems and dealing with outliers is carried out through RANSAC.

In this paper, we address the problem of registering the 3D scan and a set of images of a structured scene captured by calibrated cameras. Our assumption is that the scene is structured in the sense that it can be segmented into and represented by planes (or planar patches). Such representation is compact [2] and can also be useful for scene knowledge-based refinement methods [31. The plane-based assumption is particularly valid when dealing with man-made environments, including (but not limited to) Manhattan World, urban and indoor scenes that are abundant with planes. In our approach, we seek the metric transformation relating the scene's planes and the SfM-induced 3D structure. The SfM structure may either be represented by a sparse set of 3D points, obtained from sparse matching across images, or by planes should a sufficiently dense matching between images be obtained and the resulting point cloud segmented. When the SfM 3D structure is a sparse set of points, registration is carried out by establishing point-to-plane correspondences. Point-to-plane registration methods are known to perform better than their point-to-point counterparts 30. When the SfMinduced 3D structure consists of planes, registration is carried out by seeking plane-to-plane correspondences.

We rely on the fact that, under metric ambiguity, both point-to-plane and plane-to-plane assignments can be expressed as a second degree polynomial in scaledquaternion and translation parameters. Our approach aims at maximizing the set of inlier assignments with guaranteed optimality of the consensus set. The consensus set maximization methods [18, 1] discussed above are not applicable because of the nonlinearity of the problem at hand. In our approach, we use the Branchand-Bound $(\mathrm{BnB})$ algorithmic paradigm to explore the scaled-quaternion and translation parameter space. As in 18, 1, we rely on establishing optimistic and pessimistic sets of inlier assignments for pruning branches whose most optimistic sets are worse than the best pessimistic one. Our contribution is threefold:

(i) We propose a novel modeling of the point-to-plane (possibly point-to-patch) and plane-to-plane correspondence problems. Our modeling is based on a rigorous Sum-of-Squares polynomial optimization theory and is used to derive new conditions to identify, with certainty, mismatched correspondences within parameters' bounds. Our registration approach relies upon such conditions to build optimistic inlier assignment sets for given parameters' bounds.

(ii) We introduce SfM-specific constraints in our modeling, namely, a plane visibility criterion and optional vague constraints on the positions of the camera.

(iii) Based on our modeling and constraints, we propose a globally optimal registration algorithm that maximizes the inlier set of either point-to-plane or planeto-plane assignments in presence of putative correspondences along with its non-combinatorial counterpart in the absence of such correspondences. 
Our paper is organized as follows: Section 2 provides the main results from polynomial SoS optimization theory that we exploit in our registration approach. In Section 3 we present our working hypotheses along with the assignment polynomials arising in the point-toplane and plane-to-plane alignment problems. In Section 4. we derive polynomial SoS conditions for identifying mismatches within given registration parameters' bounds. In section 5 we present our modeling of additional geometric constraints to handle the scale ambiguity, to exploit vague knowledge on camera locations, and to perform point-to-patch rather than pointto-plane registration. Plane visibility, vague camera locations, and (to a lesser extent) scaling are rather SfMspecific constraints. These, and the point-to-patch constraints, may be used to boost the search for mismatches. This leads us to the statement of our main result that we give in Section 6 along with the description of our $\mathrm{BnB}$ registration algorithm for point-to-plane (or pointto-patch) and plane-to-plane registration. The results of our experiments are summarized and discussed in Section ??. Section 7 concludes our work.

\section{Polynomial Sum-of-Squares theory}

In this section, we present an overview of some important results in Polynomial Sum-of-Squares optimization theory. These results account for the main ingredients of our registration approach.

Definition 1 (SoS and PSD) Let $\mathbb{R}[\mathrm{x}]$ be the ring of polynomials in $n$ variables, $\mathrm{x}=\left(x_{1}, x_{2}, \ldots, x_{n}\right)$, with real-valued coefficients. A polynomial $f(\mathrm{x}) \in \mathbb{R}[\mathrm{x}]$ is

- Positive Semi-Definite (PSD) (or nonnegative) if $f(\mathrm{x}) \geq$ 0 for all $\mathrm{x} \in \mathbb{R}^{n}$;

- Sum-of-Squares (SoS) if there exist polynomials $f_{i}(\mathrm{x}) \in$ $\mathbb{R}[\mathrm{x}]$ such that $f(\mathrm{x})=\sum_{i} f_{i}(\mathrm{x})^{2}$.

A SoS is obviously always PSD and the converse is generally untrue. However, Hilbert [16 proved that, for some classes of polynomials including quadratic ones, a polynomial is PSD if and only if it is SoS. Checking whether a polynomial is PSD is NP-hard (though decidable) while checking whether a polynomial is SoS is computationally tractable using Semi-definite Programming (SDP) and employing the so-called Gram matrix of the polynomial.

Definition 2 (Gram matrix [25]) Consider a polynomial $f(\mathrm{x}) \in \mathbb{R}[\mathrm{x}]$ of degree $2 d$. Let $\mathbf{Z}_{d}(\mathrm{x})$ be the vector of monomials of $f(\mathrm{x})$ up to monomials of degree $d$. The matrix $\mathrm{G}$ such that $f(\mathrm{x})=\mathbf{Z}_{d}(\mathrm{x})^{\top} \mathrm{G} \mathbf{Z}_{d}(\mathrm{x})$ is a Gram matrix of $f(\mathrm{x})$.
Theorem $1([\mathbf{6}, \mathbf{2 5}])$ A polynomial $f(\mathrm{x}) \in \mathbb{R}[\mathrm{x}]$ of $d e-$ gree $2 d$ is SoS if and only if there exists a real symmetric positive semi-definite Gram matrix of $f(\mathrm{x})$.

Note that since odd-degree polynomials cannot be SoS, only even-degree polynomials are concerned by such test. Checking for the existence of a positive semi-definite Gram matrix $G$ boils down to solving a Linear Matrix Inequality (LMI) feasibility problem. LMI feasibility can be efficiently solved using the interior-point algorithm $[3$. Theorem 1 allows us to check whether a polynomial $f(\mathrm{x})$ is nonnegative for every $\mathrm{x} \in \mathbb{R}^{n}$. One is often interested in checking whether $f(x)$ is nonnegative in a semi-algebraic set $\mathcal{K}$ defined by polynomials $g_{i}(\mathrm{x}) \in \mathbb{R}[x]$ such that

$\mathcal{K}=\left\{\mathrm{x} \in \mathbb{R}^{n}: g_{i}(\mathrm{x}) \geq 0, i=1 \ldots m\right\}$.

This can be answered via the so-called Positivstellensatz (Psatz) 22. The Psatz states that $f(\mathrm{x})$ is nonnegative on $\mathcal{K}$ if there exist SoS polynomials $\sigma_{v}(\mathrm{x})$ such that

$f(\mathrm{x})=\sum_{v \in\{0,1\}^{m}} \sigma_{v}(\mathrm{x}) g_{1}(\mathrm{x})^{v_{1}} g_{2}(\mathrm{x})^{v_{2}} \ldots g_{m}(\mathrm{x})^{v_{m}}$.

Exploiting Psatz is difficult and may turn numerically intractable in practice because (2) requires $2^{m} \operatorname{SoS} \sigma_{v}$ polynomials. Putinar [26] provides a much simpler Psatz under Archimedean conditions on the so-called quadratic module.

Definition 3 (Quadratic module [35]) The quadratic module $M(g)=M\left(g_{1}, \ldots, g_{m}\right) \subset \mathbb{R}[x]$ of polynomials $g_{1}(\mathrm{x}), g_{2}(\mathrm{x}), \ldots, g_{m}(\mathrm{x})$ is the set

$M(g)=\left\{\sigma_{0}(\mathrm{x})+\sum_{i=1}^{m} \sigma_{i}(\mathrm{x}) g_{i}(\mathrm{x}):\right.$ each $\sigma_{i}$ is $\left.\operatorname{SoS}\right\}$.

Definition 4 (Archimedean [35]) The quadratic module $M(g)$ of polynomials $g_{1}(\mathrm{x}), g_{2}(\mathrm{x}), \ldots, g_{m}(\mathrm{x})$ is Archimedean if $N-\sum_{k=1}^{n} x_{k}^{2} \in M(g)$ for some $N \in \mathbb{N}$.

Theorem 2 (Putinar's Positivstellensatz [26]) Assume the quadratic module $M(g)$ is Archimedean. If $f(x)>0$ on $\mathcal{K}$ (defined by (1)), then $f(x) \in M(g)$.

\section{Assignment polynomials}

In this paper, we consider a set of two or more calibrated cameras observing a scene consisting of a set $\mathcal{P}$ of at least four distinct planes in general positions. The scene has been scanned by a 3D sensor and segmented into these planes. A plane $\Pi \in \mathcal{P}$ is given by its normal 3 -vector $\pi$ and signed distance to the origin $d$. We also consider the set $\mathcal{Y}$ of seven or more points (lying on at 
least four distinct scene planes) whose projections are matched across two or more cameras. We distinguish two working hypotheses depending on whether image correspondences are sparse or dense:

- when the set $\mathcal{Y}$ is sparse, the SfM-induced [15] 3D points are likewise sparse. Each reconstructed point is then represented by a $y \in \mathbb{R}^{3}$ of cartesian coordinates;

- should $\mathcal{Y}$ be sufficiently dense, the SfM-induced point cloud may further be segmented into a set of planes $\mathcal{P}_{r}$. Each such plane is represented by its normal 3 -vector $\pi_{r}$ and distance to the origin $d_{r}$.

The coordinates of the SfM-reconstructed points and/or planes and those of the scanned scene planes are represented in two distinct reference frames. The two representations of the scene also differ by a generally unknown scale factor. Consequently, the transformation aligning the SfM-reconstructed scene (whether represented by points or by planes) and the scanned scene is represented by a $3 \times 3$ scaled-rotation matrix $Q$ and a translation 3-vector t. A quaternion representation with no enforcement of unit quaternion $\mathbf{q}=(z u v w)^{\top}$ is used to represent the scaled-rotation matrix $Q$ :

$\mathbf{Q}=\left[\begin{array}{ccc}z^{2}+u^{2}-v^{2}-w^{2} & 2 u v-2 w z & 2 u w+2 v z \\ 2 u v+2 w z & z^{2}-u^{2}+v^{2}-w^{2} & 2 v w-2 u z \\ 2 u w-2 v z & 2 v w+2 u z & z^{2}-u^{2}-v^{2}+w^{2}\end{array}\right]$.

Aligning the SfM and scanned representations consists in finding $\mathrm{Q}$ and $\mathrm{t}$ that together map the 3D SfMreconstructed points, or alternatively the SfM-induced planes, to their corresponding planes in the scanned scene. The problem of finding the correct matches between the 3D SfM-reconstructed points and scanned planes is referred to as the "point-to-plane" assignment problem. That of finding the correct match between the SfM and scanned planes is referred to as the plane-toplane assignment problem. In both cases, the assignments are described by polynomials in the unknown entries of $\mathrm{Q}$ and $\mathrm{t}$ and which we denote by a vector $x \in \mathbb{R}^{7}$ such that $x=\left(q^{\top}, t^{\top}\right)^{\top}$.

Point-to-plane assignment polynomials: When relying on point-to-plane assignments for registering the image and scanner modalities, we consider $\mathcal{A} \subset \mathcal{Y} \times \mathcal{P}$ as a set of putative point-to-plane assignments $(\times$ refers to the cartesian product) and $a=(Y, \Pi) \in \mathcal{A}$ is one such assignment. The polynomial $f_{a}(\mathrm{x})$ in $\mathbb{R}[\mathrm{x}]$ induced by $a$ is given by:

$f_{a}(\mathrm{x}):=\pi^{\top}(\mathrm{Q} \mathrm{y}+\mathrm{t})-d$.

If $x$ is the true registration parameter vector, then for every correct assignment $a \in \mathcal{A}, f_{a}(\mathrm{x})=0$.
Plane-to-plane assignment polynomials: When carrying out plane-to-plane registration, the set of putative assignments $\mathcal{A}$ represents a subset of $\mathcal{P}_{r} \times \mathcal{P}$. The assignment $a=\left(\Pi_{r}, \Pi\right) \in \mathcal{A}$ is described by a 4 -vector of polynomials

$f_{a}(\mathrm{x}):=\mathrm{q}^{\top} \mathrm{q}\left[\begin{array}{c}\pi_{r} \\ -d_{r}\end{array}\right]+\delta\left[\begin{array}{ll}\mathrm{Q}^{\top} & 0 \\ \mathrm{t}^{\top} & 1\end{array}\right]\left[\begin{array}{c}\pi \\ -d\end{array}\right]$.

Should $x$ be the true registration parameter vector, all four polynomials in $f_{a}(\mathrm{x})$ simultaneously vanish for some value of $\delta= \pm 1$.

\section{Polynomial SoS assignment conditions}

Our goal is to simultaneously estimate the registration parameters $x$ and associated set of correct assignments. The problem can be solved by considering either pointto-plane or plane-to-plane assignments. Note, however, that we voluntarily do not distinguish between the cases in which the initial set $\mathcal{A}$ is a point-to-plane putative assignments set from that in which it is a plane-to-plane one. Unless stated otherwise, $f_{a}(\mathrm{x})$ is always considered as a vector of polynomials of appropriate dimension: a 1-vector representing the single polynomial (4) induced by a point-to-plane putative assignment or a 4 -vector of polynomials (5) induced by a plane-to-plane assignment. $f_{a}(\mathrm{x})=0$ means that all polynomials in this vector simultaneously vanish. We may assume, for the sake of clarity of the exposition, that, in the case of plane-toplane assignments, the value of $\delta= \pm 1$ in (4) is known. It will be made clear, further in this section (see Result 3), how the two cases are handled in our method.

Our registration approach is based on the BnB algorithmic paradigm and branching is carried out on the space of registration parameters $x$. At each iteration, we are given parameter intervals, in the form of two vectors $\underline{x}$ and $\overline{\mathrm{x}}$ in $\mathbb{R}^{7}$ whose respective entries $\underline{x}_{k}$ and $\bar{x}_{k}$ satisfy $\underline{x}_{k} \leq \bar{x}_{k}$ for $k=1 \ldots 7$. Although the full approach is detailed further in the paper, the idea is that such intervals are to be probed for potentially correct assignments by attempting to solve the following problem:

Problem 1 For a given $a \in \mathcal{A}$, is there a vector $\mathrm{x} \in \mathbb{R}^{7}$ satisfying $\underline{x}_{k} \leq x_{k} \leq \bar{x}_{k}, k=1 \ldots 7$ such that $f_{a}(\mathrm{x})=$ $0 ?$

In other words, one would like to know whether all polynomials in $f_{a}(\mathrm{x})$ cross zero for some $\mathrm{x}$ within the considered bounds. The assignment would then qualify as a potential inlier, i.e. possible correct assignment, within these bounds. This is however difficult to answer. Considering only one polynomial or attempting to establish 
whether the polynomials in $f_{a}(\mathrm{x})$ cross zero for possibly different values of $x$ within the bounds may seem less demanding. While doing so wouldn't make the problem any easier, considering the following alternative does:

Problem 2 For a given $a \in \mathcal{A}$, is there a vector $\lambda_{a}$ of scalars of appropriate dimension such that $\lambda_{a}^{\top} f_{a}(\mathrm{x})>0$ for every $\mathrm{x}$ satisfying $\underline{x}_{k} \leq x_{k} \leq \bar{x}_{k}$ for $k=1 \ldots 7$ ?

If $\lambda_{a}^{\top} f_{a}(\mathrm{x})>0$, then at least one of the polynomials in $f_{a}(\mathrm{x})$ is guaranteed not to cross zero. As a result, the assignment $a$ is definitely an outlier, i.e. incorrect assignment, within the bounds. Otherwise, it is a potential inlier. Indeed, if the question of Problem 2 is answered in the affirmative, the one of Problem 11 is answered in the negative: i.e. there exist no $x$ in the interval with which $f_{a}(\mathrm{x})=0$. Furthermore, one can rely on Putinar's Theorem 2 to solve Problem 2 . To do so, assume we are given a set of polynomials $g_{i}(\mathrm{x})$ whose quadratic module $M(g)$ is Archimedean: if, for $\lambda_{a}$ a vector of scalars of appropriate dimension, $\lambda_{a}^{\top} f_{a}(\mathrm{x})>0$ for all $\mathrm{x} \in \mathcal{K}=\left\{\mathrm{x} \in \mathbb{R}^{7}: g_{i}(\mathrm{x}) \geq 0, i=1 \ldots m\right\}$, then $\lambda_{a}^{\top} f_{a}(\mathrm{x}) \in M(g)$. Hence, there must exist SoS polynomials $\sigma_{i}$ such that:

$\lambda_{a}^{\top} f_{a}(\mathrm{x})-\sum_{i=1}^{m} \sigma_{i}(\mathrm{x}) g_{i}(\mathrm{x})$ is $\operatorname{SoS}$.

Note that, in general, if $(6)$ is satisfied, then $\lambda_{a}^{\top} f_{a}(\mathrm{x})$ may not be necessarily positive in $\mathcal{K}$ since $\mathcal{K}$ could possibly be empty. However, so long as $\mathcal{K}$ is not empty and $\sigma_{i}$ SoS polynomials can be found, one is guaranteed that $\lambda_{a}^{\top} f_{a}(\mathrm{x})>0$ everywhere in $\mathcal{K}$ since $\sum_{i=1}^{m} \sigma_{i}(\mathrm{x}) g_{i}(\mathrm{x})>0$ in $\mathcal{K}$.

There are two main pending issues before one is able to use (6). First, one needs to find a set of polynomials $g_{i}(\mathrm{x})$, representative of the parameter intervals, whose quadratic module $M(g)$ is Archimedean. Second, it is so far unclear how the $\sigma_{i}$ SoS polynomials can be found. Let us explore now the first of these issues. Note that the Archimedean property is a matter of representation and the quadratic module of the set constructed from the linear interval constraints $x_{k}-\underline{x}_{k} \geq 0$ and $\bar{x}_{k}-x_{k} \geq 0$ is not Archimedean. In the following, we show that quadratic polynomial inequalities derived from such bound constraints yield an Archimedean quadratic module.

Proposition 1 Consider the polynomials $g_{k}(\mathrm{x})=\left(x_{k}-\right.$ $\left.\underline{x}_{k}\right)\left(\bar{x}_{k}-x_{k}\right), k=1 \ldots 7$. The quadratic module $M(g)$ of these polynomials is Archimedean.

Proof As per Definition 4 for $M(g)$ to qualify as Archimedean, one ought to demonstrate that $N-\sum_{k=1}^{n} x_{k}^{2} \in$ $M(g)$ for some $N \in \mathbb{N}$. Definition 3 indicates that, for
$N-\sum_{k=1}^{n} x_{k}^{2}$ to be in $M(g)$ with the $g_{k}(\mathrm{x})$ polynomials of this proposition, there must exist SoS polynomials $\sigma_{0}(\mathrm{x})$ and $\sigma_{k}(\mathrm{x}), k=1 \ldots 7$, such that

$N-\sum_{k=1}^{n} x_{k}^{2}=\sigma_{0}(\mathrm{x})+\sum_{k=1}^{7} \sigma_{k}(\mathrm{x})\left(x_{k}-\underline{x}_{k}\right)\left(\bar{x}_{k}-x_{k}\right)$.

In particular, because $\sigma_{0}(\mathrm{x})$ must be SoS, the proof boils down to establishing the existence of SoS $\sigma_{k}(\mathrm{x}), k=$ $1 \ldots 7$ that can turn $\sigma_{0}(\mathrm{x})$, of the form

$\sigma_{0}(\mathrm{x})=N-\sum_{k=1}^{7} \mathrm{x}_{k}^{2}-\sum_{k=1}^{7} \sigma_{k}(\mathrm{x})\left(x_{k}-\underline{x}_{k}\right)\left(\bar{x}_{k}-x_{k}\right)$,

into a SoS polynomial. The polynomial $\sigma_{0}(\mathrm{x})$ can be rewritten as

$\sigma_{0}(\mathrm{x})=N-\sum_{k=1}^{7}\left(x_{k}^{2}+\sigma_{k}(\mathrm{x})\left(x_{k}-\underline{x}_{k}\right)\left(\bar{x}_{k}-x_{k}\right)\right)$.

Let us first show that a PSD, not necessarily SoS, $\sigma_{0}(\mathrm{x})$ exists. To do so, we thus seek $\sigma_{k}(\mathrm{x}), k=1 \ldots 7$, for which there exists $N$ satisfying

$N \geq \max _{\times}\left(\sum_{k=1}^{7}\left(x_{k}^{2}+\sigma_{k}(\mathrm{x})\left(x_{k}-\underline{x}_{k}\right)\left(\bar{x}_{k}-x_{k}\right)\right)\right)$.

Such $N$ exists if the polynomial argument of $\max ($.$) is$ concave. Observe that using zero-degree SoS polynomials $\sigma_{k}$ that are independent from x, i.e. nonnegative real scalars, this polynomial is quadratic. Its expansion into

$\sum_{k=1}^{7}\left(\left(1-\sigma_{k}\right) x_{k}^{2}+\sigma_{k}\left(\underline{x}_{k}+\bar{x}_{k}\right) x_{k}-\sigma_{k} \underline{x}_{k} \bar{x}_{k}\right)$

shows that its Hessian matrix, $\mathrm{H}=\operatorname{diag}\left(\left(1-\sigma_{1}\right),(1-\right.$ $\left.\left.\sigma_{2}\right), \ldots,\left(1-\sigma_{7}\right)\right)$, is diagonal. This polynomial is then concave if $\mathrm{H}$ is negative-definite which happens for $\sigma_{k}>$ 1 for $k=1 \ldots 7$. This shows that $N$ and nonnegative scalars $(\mathrm{SoS}) \sigma_{k}, k=1 \ldots 7$, do exist for $\sigma_{0}(\mathrm{x})$ to be PSD. Furthermore, $\sigma_{k}$ being scalars, $\sigma_{0}(\mathrm{x})$ is a quadratic polynomial. As discussed in Section 2, Hilbert [16] showed that, for quadratic polynomials, every PSD polynomial is SoS.

Let us now consider the problem of checking whether or not [6] is SoS when considering the polynomials $g_{k}(\mathrm{x}), k=1 \ldots 7$ of Proposition 1. If so the assignment $a$ is definitely an outlier within the bounds. If one knows beforehand that $\lambda_{a}^{\top} f_{a}(\mathrm{x})$ must be positive, a sequence of $\sigma_{k}(\mathrm{x})$ of increasing degree can be used until a positivity certificate is obtained. However, for the problem at hand, when a set of $\sigma_{k}(\mathrm{x})$ of some degree fails to deliver such certificate, it is either because $\lambda_{a}^{\top} f_{a}(\mathrm{x})$ 
indeed crosses zero (inlier) or the required degree for a positivity certificate has not been reached. The good news here is that, within a $\mathrm{BnB}$ search, the considered bound intervals $[\underline{x}, \bar{x}]$ get smaller and we show in the following that using nonnegative scalars $\sigma_{k}$ rather than SoS polynomials of higher degree suffices. To see this, consider the following proposition:

Proposition 2 Let $\hat{x} \in \mathbb{R}^{7}$ with known entries. The following statements are equivalent

(i) $\lambda_{a}^{\top} f_{a}(\hat{\mathrm{x}})>0$.

(ii) $\exists$ nonnegative scalars $\sigma_{k} \in \mathbb{R}, k=1 \ldots 7$ :

$\lambda_{a}^{\top} f_{a}(\mathrm{x})+\sum_{k=1}^{7}\left(\mathrm{x}_{k}-\hat{\mathrm{x}}_{k}\right)^{2} \sigma_{k}>0$.

Proof (ii) $\Longrightarrow$ (i) is straightforward.

For (i) $\Longrightarrow$ (ii), consider $\lambda_{a}^{\top} f_{a}(\mathrm{x})$ 's Gram matrix $\mathrm{G}_{f}$ and $\mathrm{G}_{\mathrm{x}}$ that of $\sum_{k=1}^{7}\left(\mathrm{x}_{k}-\hat{\mathrm{x}}_{k}\right)^{2}$. These matrices are defined by: $f_{a}(\mathrm{x})=\mathrm{x}^{\top} \mathrm{G}_{f} \mathrm{x}$ and $\sum_{k=1}^{7}\left(\mathrm{x}_{k}-\hat{\mathrm{x}}_{k}\right)^{2}=\mathrm{x}^{\top} \mathrm{G}_{\mathrm{x}} \mathrm{x}$. Note that $G_{x}$ is PSD and can be written as $G_{x}=U^{\top} U$ with $U \hat{x}=0$. The Gram matrix of the polynomial in $\sqrt{12}$ is then written as $\mathrm{G}_{f}+\mathrm{U}^{\top} \operatorname{diag}\left(\sigma_{1}, \sigma_{2}, \ldots, \sigma_{7}\right) \mathrm{U}$. A direct application of Finsler's lemma 11 is that the latter matrix is positive-definite if and only if $\hat{x}^{\top} G_{f} \hat{x}>0$. This, not only shows $(\mathrm{i}) \Longrightarrow$ (ii), but also proves the equivalence of the two statements.

We now state the following preliminary result:

Result 3 (Preliminary) Consider two vectors $\underline{x}$ and $\overline{\mathrm{x}}$ in $\mathbb{R}^{7}$ whose respective entries $\underline{\mathrm{x}}_{k}$ and $\overline{\mathrm{x}}_{k}$ satisfy $\underline{\mathrm{x}}_{k} \leq$ $\overline{\mathrm{x}}_{k}$ for $k=1 \ldots 7$. Let $\mathcal{K}_{b}$ be the set

$\mathcal{K}_{b}=\left\{\mathrm{x} \in \mathbb{R}^{7}: g_{k}(\mathrm{x}):=\left(\mathrm{x}_{k}-\underline{\mathrm{x}}_{k}\right)\left(\overline{\mathrm{x}}_{k}-\mathrm{x}_{k}\right) \geq 0\right\}$,

$a \in \mathcal{A}$ be either a point-to-plane or plane-to-plane putative assignment, and $f_{a}(\mathrm{x})$ the vector of polynomials induced by this assignment (4) for point-to-plane and (5) for plane-to-plane).

If $\exists$ a vector $\lambda_{a}$ of appropriate dimension and nonnegative scalars $\sigma_{k}$ such that

$\lambda_{a}^{\top} f_{a}(\mathrm{x})-\sum_{k=1}^{7} g_{k}(\mathrm{x}) \sigma_{k}$

is $S o S$, then $\lambda_{a}^{\top} f_{a}(\mathrm{x})>0$ for every $\underline{\mathrm{x}}_{k} \leq \mathrm{x}_{k} \leq \overline{\mathrm{x}}_{k}$. In this case, one of the polynomials in $f_{a}(\mathrm{x})$ never crosses zero within the considered bounds of $\mathrm{x}$. The assignment a is then guaranteed to be an outlier (a point-to-plane or plane-to-plane mismatch) within these bounds. Otherwise, a is a potential inlier. Note that, in the case of a plane-to-plane assignment a, the latter is deemed an outlier only if (14) is found to be SoS for both values \pm 1 of $\delta$ in $\sqrt{5}$.
Furthermore, a consequence of Proposition 2 is that when $\overline{\mathrm{x}}_{k}-\underline{\mathrm{x}}_{k}$ tends towards zero, we are guaranteed that any outlier within the bound is detected. Indeed, this can be seen by noticing that when $\overline{\mathrm{x}}_{k}=\underline{\mathrm{x}}_{k}=\hat{\mathrm{x}}_{k}$, polynomial (14) turns into (12).

Whether (14) is SoS can be tested by converting it into its corresponding Gram matrix LMI feasibility problem for the $\lambda_{a}$ and $\sigma_{k}$ indeterminates. Although the guarantee of identifying outliers using scalar $\sigma_{k}$ multipliers is demonstrated with a zero-gap bound, in practice, outliers are detected very early in the process. As demonstrated in our experiments, the ability to detect outliers is improved with every size reduction of the investigated bounds. It may be tempting to use higher degree $\sigma_{k}(\mathrm{x})$ SoS polynomials to boost the process. However, this is unnecessary and yields slower performances compared to branching.

\section{Semi-algebraic sets for geometric constraints}

Recall that our goal is to register a SfM-induced reconstruction of points or planes and a plane-segmented scanned scene. Unlike when dealing with 3D-3D registration, additional geometric constraints emanating from the cameras can be exploited. Some may be implicit, such as plane visibility, others, such as vague camera locations, may be obtained from extra knowledge. In addition, when dealing with segmented scanned scenes, one is given planar patches rather than infinite planes. These patches can be used for point-to-patch in lieu of point-to-plane registration to restrict the location of the SfM-induced points patches. In all cases, these constraints are described by quadratic polynomial inequalities that may augment the semi-algebraic set $\mathcal{K}_{b}$ derived from the bound constraints. Indeed, adding new polynomial inequalities to those in $\mathcal{K}_{b}$ has no effect on the Archimedean property of its quadratic module and Proposition 2 still holds. Note though that some of the constraints presented herein, which we refer to as "generic constraints", may be exploited in both pointto-plane and plane-to-plane registration. Others are applicable only to point-to-plane registration.

\subsection{Generic constraints}

Whether engaging in point-to-plane or plane-to-plane, extra-knowledge about vague camera locations or scale information can be used as additional registration constraints.

Camera bounds: A camera center $C$ may lie within a box delimited by six planes in the set $\Psi=\left\{\Psi_{k}\right\}_{k=1}^{6}$ 
defined by their normal vectors $\psi_{k}$ and signed distances $d_{k}$. Such information can be obtained from applicationspecific knowledge (GPS, moving vehicle, etc.). This knowledge can be used for further enforcing the search for point-to-plane or plane-to-plane outliers and turns very useful when no putative correspondences are initially known. Consider the cartesian coordinate vector $\mathrm{c}$ of the camera center and let

$$
\begin{aligned}
\mathcal{K}_{c}= & \left\{\mathrm{x} \in \mathbb{R}^{7}: h_{k}(\mathrm{x}):=\left(\psi_{k}^{\top}(\mathrm{Q} \mathrm{c}+\mathrm{t})-d_{k}\right) \delta_{k} \geq 0,\right. \\
& k=1 \ldots 6\}
\end{aligned}
$$

where $\delta_{k}$ is the known sign, with respect to $\Phi_{k}$, of any point within the considered box. If $h_{k}(\mathrm{x})$ are positive, the camera center is within the box. One can now test if $\lambda_{a}^{\top} f_{a}(\mathrm{x})>0$ whenever the camera center is in the box defined by $\mathcal{K}_{c}$.

Quaternions and scale: In the absence of scale, quaternion parameters demand that $\mathrm{q}^{\top} \mathrm{q}=1$. When dealing with a scaled scene, the rotation is represented by a scaled quaternion matrix and one can only enforce that $q^{\top} q>0$. It is understood that, in order to keep the problem numerically tractable via the Archimedean property, all registration parameters need to be bounded. The scale of the scene is no exception. When a better lower bound $\underline{s}>0$ on the scale $s$ is available, it is advised to enforce that $\mathrm{q}^{\top} \mathrm{q} \geq s$. This condition does not appear in the set $\mathcal{K}_{b}$ and hence must be accounted for. Assuming the entries $\mathrm{x}_{k}, k=1 \ldots 4$ of $\mathrm{x}$ correspond the quaternion parameters, we consider the set

$\mathcal{K}_{q}=\left\{\mathrm{x} \in \mathbb{R}^{7}: q(\mathrm{x}):=-\underline{s}+\sum_{k=1}^{4} \mathrm{x}_{k}^{2} \geq 0\right\}$

Furthermore, since both $\mathrm{q}$ and $-\mathrm{q}$ yield the same rotation matrix, the initial lower bound of one of the quaternion parameters may arbitrarily be chosen nonnegative. The rest of the quaternion parameters may be initially bounded between $-\sqrt{\bar{s}}$ and $\sqrt{\bar{s}}$ where $\bar{s}$ is the scale's upper bound.

\subsection{Additional point-to-plane geometric constraints}

In addition to camera bounds and scale constraints, other constraints can be used when dealing specifically with point-to-plane registration.

Patches: Consider a plane $\Pi$, from the scanned scene, and three or more planes $\Phi_{k}$, not necessarily from the scene, orthogonal to it. The $\Phi_{k}$ planes must be chosen such that their intersection with $\Pi$ defines a convex region on $\Pi$. The set of points on $\Pi$ within this convex region is a patch. In practice, four such planes are adequate to represent meaningful patches in manmade environments. Each $\Phi_{k}$ is described by its normal vector $\phi_{k}$ and signed distance $d_{k}$. Let us denote by $\Phi$ the set $\left\{\Phi_{k}\right\}_{k=1}^{4}$ and let $\delta_{k}= \pm 1$ be the known sign, with respect to $\Phi_{k}$, of a scanned point lying within the considered region. In the point-to-patch case, we can then identify outliers by checking whether point-toplane $f_{a}(\mathrm{x})$ (4) is positive everywhere within $\mathrm{x}^{\prime} s$ bounds and in the set

$$
\begin{aligned}
\mathcal{K}_{a}^{\Phi}= & \left\{\mathrm{x} \in \mathbb{R}^{7}: p_{k}(\mathrm{x}):=\left(\phi_{k}^{\top}(\mathrm{Q} \mathrm{y}+\mathrm{t})-d_{k}\right) \delta_{k} \geq 0,\right. \\
& k=1 \ldots 4\}
\end{aligned}
$$

The polynomials in this set indicate the sign of the point $Y$, with coordinates $\mathrm{y}$, with respect to each $\Phi_{k}$.

Plane visibility: Consider a point $Y$ on a scene plane $\Pi$. If this point is imaged by two cameras, then these can only observe the same side of the plane: the one on which the point lies. In order for the cameras to observe the same side of the plane, their camera centers must lie on one side with respect to $\Pi$. Camera centers can easily be obtained from the SfM-calculated camera matrices: they are their right null space. Let $C_{k}$ be the camera centers of $n \geq 2$ cameras with cartesian coordinates $\mathrm{c}_{k}$. We define the set $\mathcal{K}_{\Pi}^{\delta}$ such that

$$
\begin{aligned}
\mathcal{K}_{\Pi}^{\delta}= & \left\{\mathrm{x} \in \mathbb{R}^{7}: v_{k}(\mathrm{x}):=\left(\pi^{\top}\left(\mathrm{Qc}_{k}+\mathrm{t}\right)-d\right) \delta \geq 0,\right. \\
& k=1 \ldots n\}
\end{aligned}
$$

where $\delta= \pm 1$. We denote $\mathcal{K}_{\Pi}^{+}$the set $\mathcal{K}_{\Pi}^{\delta}$ obtained using $\delta=+1$ and $\mathcal{K}_{\Pi}^{-}$otherwise. A given assignment $a$ is a definite outlier if $f_{a}(\mathrm{x})>0$ in $\mathcal{K}_{\Pi \Pi}^{+}$and in $\mathcal{K}_{\Pi}^{-}$(in addition to patch and bounds conditions). Furthermore, planes for which $v_{1}(\mathrm{x})$ and $v_{2}(\mathrm{x})$ (for two cameras 1 and 2) always have opposite signs within $x^{\prime} s$ bounds cannot be assigned any points visible in those cameras. This would indicate that the plane always cuts the base-line of the two camera and cannot contain points visible in both cameras. Testing this can be carried out by checking, for $\delta= \pm 1$, whether

$$
\left\{\begin{array}{l}
\exists \sigma_{k}: v_{1}(\mathrm{x})-\sum_{k=1}^{7} g_{k}(\mathrm{x}) \sigma_{k} \text { is } S o S, \\
\exists \sigma_{k}:-v_{2}(\mathrm{x})-\sum_{k=1}^{7} g_{k}(\mathrm{x}) \sigma_{k} \text { is } S o S .
\end{array}\right.
$$

If for both values of $\delta$, each polynomial in 19 is SoS, plane $\Pi$ shall not be considered for assigning SfM points emanating from those cameras.

\section{Registration}

The preliminary results in Result 3 apply to both pointto-patch and plane-to-plane registration problems. Based on these results and the semi-algebraic sets presented in 
Section 5, we are now ready to state additional results for point-to-plane, or rather plane-to-patch, and planeto-plane registration. These results along with those in Result 3 are used in our $\mathrm{BnB}$ registration algorithm which is also presented in this Section.

Result 4 (Point-to-patch) Assume we are given a putative point-to-plane assignment $a=(Y, \Pi) \in \mathcal{A}$, a patch on $\Pi$ delimited by the planes in the set $\Phi=$ $\left\{\Phi_{k}\right\}_{k=1}^{4}$, lower $\underline{x}$ and upper $\bar{x}$ bounds on the registration parameter vector $\mathrm{x}$, bounds $\underline{s}$ and $\bar{s}$ on the scale of the scene, and (optionally) bounds defined by planes $\Psi=\left\{\Psi_{k}\right\}_{k=1}^{6}$ on the location of the camera centers of one (possibly more) camera. One would like to know whether or not the SfM-reconstructed point Y may lie on $\Pi$, while $\Pi$ is visible by the cameras observing $Y$, within the patch $\Phi$ with registration parameters in the bounds $\underline{\mathrm{x}}$ and $\overline{\mathrm{x}}$. In order to establish whether such assignment is possible, we consider the set

$\mathcal{K}=\left\{\mathrm{x} \in \mathbb{R}^{7}: \mathrm{x} \in \mathcal{K}_{b} \cap \mathcal{K}_{a}^{\Phi} \cap \mathcal{K}_{\Pi}^{\delta} \cap \mathcal{K}_{c} \cap \mathcal{K}_{q}\right)$

resulting from the intersection of all the sets defined by (13), (17), (18), (15) and (16). If there exist a scalar $\lambda_{a}$ and nonnegative scalars $\sigma_{k}, \sigma_{k}^{\prime}, \sigma_{k}^{\prime \prime}, \sigma_{k}^{\prime \prime \prime}$ and $\sigma$ such that

$\lambda_{a} f_{a}(\mathrm{x})-\sum_{k=1}^{7} g_{k}(\mathrm{x}) \sigma_{k}-\sum_{k=1}^{4} p_{k}(\mathrm{x}) \sigma_{k}^{\prime}$

$-\sum_{k=1}^{n} v_{k}(\mathrm{x}) \sigma_{k}^{\prime \prime}-\sum_{k=1}^{6} h_{k}(\mathrm{x}) \sigma_{k}^{\prime \prime \prime}-q(\mathrm{x}) \sigma$

is $S O S$, then $\lambda_{a} f_{a}(\mathrm{x})>0$ in $\mathcal{K}$ and the assignment $a$ is a definite outlier. It is a potential inlier otherwise.

Note that a point-to-plane version of this results can simply be obtained by not using $\mathcal{K}_{a}^{\Phi}$ to construct $\mathcal{K}$.

Result 5 (Plane-to-plane) Assume we are given a putative plane-to-plane assignment $a=\left(\Pi_{r}, \Pi\right) \in \mathcal{A}$, lower $\underline{x}$ and upper $\overline{\mathrm{x}}$ bounds on the registration parameter vector $\mathrm{x}$, bounds $\underline{s}$ and $\bar{s}$ on the scale of the scene, and (optionally) bounds defined by planes $\Psi=\left\{\Psi_{k}\right\}_{k=1}^{6}$ on the location of the camera centers of one (possibly more) camera. One would like to know whether or not the SfM-reconstructed plane $\Pi_{r}$ may be aligned with $\Pi$ for registration parameters in the bounds $\underline{x}$ and $\overline{\mathrm{x}}$. In order to establish whether such assignment is possible, we consider the set

$\mathcal{K}=\left\{x \in \mathbb{R}^{7}: x \in \mathcal{K}_{b} \cap \mathcal{K}_{c} \cap \mathcal{K}_{q}\right)$

resulting from the intersection of all the sets defined by (13), (15) and (16). If there exist a 4-vector $\lambda_{a}$ of scalars and nonnegative scalars $\sigma_{k}, \sigma_{k}^{\prime}$ and $\sigma$ such that

$\lambda_{a}^{\top} f_{a}(\mathrm{x})-\sum_{k=1}^{7} g_{k}(\mathrm{x}) \sigma_{k}-\sum_{k=1}^{6} h_{k}(\mathrm{x}) \sigma_{k}^{\prime}-q(\mathrm{x}) \sigma$ is $S O S$, then $\lambda_{a}^{\top} f_{a}(\mathrm{x})>0$ in $\mathcal{K}$ and the assignment $a$ is a definite outlier. It is a potential inlier otherwise.

Recall that SoS problems 21) and 23) in these results can be solved as a LMI feasibility problem.

Our registration approach is based on Results 4 and 5 In the following, we use the term point-to-plane to refer to both point-to-plane and point-to-patch assignments. The goal of the $\mathrm{BnB}$ algorithm is to estimate the registration parameters yielding the largest number of inliers. Our algorithm is provided either a set of putative point-to-plane or plane-to-plane correspondences. In the absence of such correspondences, we consider every SfM-induced point or plane to be putatively assigned to all the planes (or patches) in the scanned scene. A dynamically-built search tree, whose nodes are registration parameters' bounds, allows to explore the space of parameters. Given an assignments and bounds on the registration parameters, the algorithm (see Algorithm 1) estimates the optimistic number of potential inliers using appropriate semi-algebraic sets from Results 4 and 5 . A local refinement method, explained below, is used to obtain a pessimistic number of inliers for each given node. We keep track of the highest number of potential inliers (bestPessimistic in Algorithm 1) over all bound intervals. Any node whose optimistic number of inliers is worse than bestPessimistic is rejected. Otherwise, the node is qualified and branched along its longest edge resulting in two new nodes to be processed. The node corresponding to the bestPessimistic number of inliers is processed first. The algorithm terminates when no node has an optimistic number of inliers that is better than bestPessimistic.

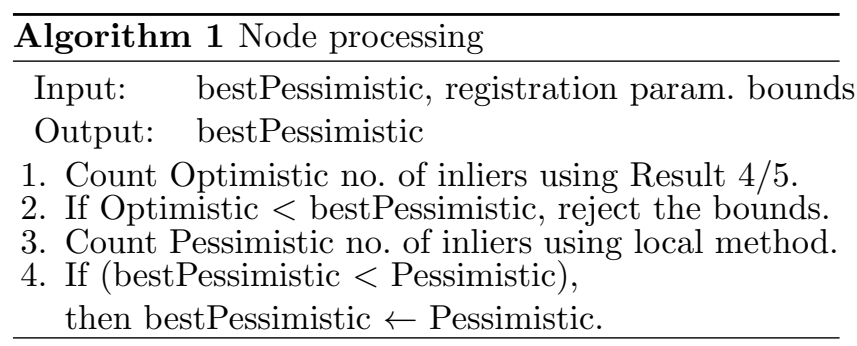

To qualify a point as an inlier, we distinguish two cases:

1. Putative point-to-plane (resp. plane-to-plane) correspondences are provided: a point-to-plane (resp. plane-to-plane) assignment qualifies as a potential inlier if (21) (resp. 23) is not proven SoS.

2. No putative correspondences are provided: the pointto-plane (resp. plane-to-plane) assignment is considered a potential inlier as soon as 21) (resp. 23) is not proven SoS. 
Local refinement: To maximize the bestPessimistic number of inliers, the local method iteratively refines the registration parameters. The refinement process starts from the mid-values of the registration parameters' bounds. In order to be representative of the node, it searches the optimal solution within the investigated bounds. Given the assignments, the algorithm iteratively updates the registration parameters to:

$\underset{\mathrm{x} \in \mathcal{K}_{b}}{\operatorname{argmin}} \sum_{a \in \mathcal{A}}\left\|f_{a}(\mathrm{x})\right\|^{2}$

At each iteration, only those assignments with $\left|f_{a}(\mathrm{x})\right|<$ $\xi$ are selected for some threshold $\xi$. In case of insufficient number of assignments, the refinement process is terminated with the best obtained output so far.

Discussion: In general, our method converges while the explored bounds are still quite large. The solution maximizing the inlier consensus set is the one returned by the local method. When the bounds are large enough, polynomials constructed from noisy data would still cross zero within the bounds allowing inliers, although affected by noise, to be accounted for. Therefore, the robustness to noise is more influenced by the local method than it is by the SoS tests. In our implementation, no special care was taken to further deal with noise when using SoS tests. However, in the case of highly noisy data, the proposed SoS framework may allow to deal more efficiently with noise by incorporating an extra bounded variable $\epsilon$ (bounded by the allowed threshold), accounting for noise. For example, if the point-to-plane assignment polynomial $f_{a}(\mathrm{x})$ does not cross zero at the sought solution, $f_{a}(\mathrm{x})+\epsilon$ (for some value of $\epsilon$ ) would. Furthermore, we have assumed throughout that the camera information fed to our algorithm is, to some extent, reliable. Should incorrect/noisy information about a camera be used, it may cause, especially with small camera bounding boxes, the registration to fail. In such cases, the camera-to-box constraints may be include in the set of putative assignments when maximizing the consensus set.

\section{Conclusion}

We proposed a method for registering a 3D scan and a set of images of a structured scene. The proposed approach is based on the theory of polynomial SoS optimization. Our method uses SoS registration conditions for point-to-plane as well as plane-to-plane registration. The method presented in this paper can incorporate various constraints emanating from scene and camera knowledge (patch segmentation, camera locations, plane visibility, scaling, etc.). Using Branch-andBound and SoS theory, we devised a robust and optimal method for inlier set maximization of either point-toplane or plane-to-plane correspondences. Although the problem is nonlinear and combinatorial, our method has provided outstanding results in terms of robustness and optimality. In particular, the employed optimization framework has the potential to be efficiently applied to other nonlinear Computer Vision problems.

Acknowledgements This research has been funded by the International Project NRF-ANR DrAACaR: ANR-11-ISO30003, the Regional Council of Bourgogne and European Regional Development Fund.

\section{References}

1. J. Bazin, Hongdong Li, In So Kweon, C. Demonceaux, P. Vasseur, and K. Ikeuchi. A branch-and-bound approach to correspondence and grouping problems. In IEEE Transactions on Pattern Analysis and Machine Intelligence (PAMI), pages 1565-1576, 2013.

2. Dorit Borrmann, Jan Elseberg, Kai Lingemann, and Andreas Nüchter. The 3d hough transform for plane detection in point clouds: A review and a new accumulator design. In 3D Research, pages 32:1-32:13, 2011.

3. Stephen Boyd and Lieven Vandenberghe. Convex Optimization. Cambridge University Press, New York, NY, USA, 2004.

4. Manmohan Chandraker, Sameer Agarwal, Fredrik Kahl, David Nister, and David Kriegman. Autocalibration via rank-constrained estimation of the absolute quadric. In IEEE Conference on Computer Vision and Pattern Recognition (CVPR), pages 1-8, 2007.

5. G. Chesi, A. Garulli, A. Vicino, and R. Cipolla. Estimating the fundamental matrix via constrained least-squares: a convex approach. IEEE Transactions on Pattern Analysis and Machine Intelligence (PAMI), pages 397-401, March 2002.

6. M.D. Choi, T.Y Lam, and B. Reznick. Sums of squares of real polynomials. Proceedings of Symposia in Pure Mathematics, 2(58):103-126, 1995.

7. Stéphane Christy and Radu Horaud. Iterative pose computation from line correspondences. In Computer Vision and Image Understanding (CVIU), pages 137-144, January 1999.

8. M. Corsini, M. Dellepiane, F. Ganovelli, R. Gherardi, A. Fusiello, and R. Scopigno. Fully automatic registration of image sets on approximate geometry. International Journal of Computer Vision (IJCV), pages 91111, March 2013.

9. Shaoyi Du, Nanning Zheng, Shihui Ying, Qubo You, and Yang Wu. An extension of the icp algorithm considering scale factor. In IEEE International Conference on Image Processing (ICIP), pages V-193, 2007.

10. Luis Ferraz, Xavier Binefa, and Francesc Moreno-Noguer. Very fast solution to the pnp problem with algebraic outlier rejection. In IEEE Conference on Computer Vision and Pattern Recognition (CVPR), pages 501-508, 2014. 
11. P. Finsler. Uber das vorkommen definiter und semidefiniter formen in scharen quadratischer formen. Comment. Math. Helv., 9, pages 188-192, 1936/37.

12. Martin A. Fischler and Robert C. Bolles. Random sample consensus: A paradigm for model fitting with applications to image analysis and automated cartography. In Commun. ACM, pages 381-395, 1981.

13. A. W. Fitzgibbon. Robust registration of $2 \mathrm{D}$ and $3 \mathrm{D}$ point sets. In The British Machine Vision Conference (BMVC), pages 662-670, 2001.

14. Adlane Habed, Kassem Al Ismaeil, and David Fofi. A new set of quartic trivariate polynomial equations for stratified camera self-calibration under zero-skew and constant parameters assumptions. In European Conference on Computer Vision (ECCV), pages 710-723. 2012.

15. R. I. Hartley and A. Zisserman. Multiple View Geometry in Computer Vision. Cambridge University Press, second edition, 2004.

16. David Hilbert. Uber die darstellung definiter formen als summe von formen quadraten. In Math. Ann., pages 342-350, 1888.

17. Jean B. Lasserre. Global optimization with polynomials and the problem of moments. SIAM J. on Optimization, pages 796-817, 2000.

18. Hongdong Li. Consensus set maximization with guaranteed global optimality for robust geometry estimation. In IEEE International Conference on Computer Vision (ICCV), pages 1074-1080, 2009.

19. Lingyun Liu and Ioannis Stamos. Automatic $3 \mathrm{~d}$ to $2 \mathrm{~d}$ registration for the photorealistic rendering of urban scenes. In IEEE Conference on Computer Vision and Pattern Recognition (CVPR), pages 137-143, 2005.

20. Andrew Mastin, Jeremy Kepner, and John W. Fisher III. Automatic registration of lidar and optical images of urban scenes. In IEEE Conference on Computer Vision and Pattern Recognition (CVPR), pages 2639-2646, 2009.

21. C. Olsson, F. Kahl, and M. Oskarsson. The registration problem revisited: Optimal solutions from points, lines and planes. In IEEE Conference on Computer Vision and Pattern Recognition (CVPR), pages 1206-1213, 2006.

22. Pablo A. Parrilo. Structured semidefinite programs and semialgebraic geometry methods in robustness and optimization. Technical report, California Institute of Technology, 2000.

23. Danda Pani Paudel, Cédric Demonceaux, Adlane Habed, and Pascal Vasseur. Localization of $2 \mathrm{~d}$ cameras in a known environment using direct $2 \mathrm{~d}$ - 3 d registration. In International Conference on Pattern Recognition (ICPR), pages 1-6, 2014.

24. Tobias Plotz and Stefan Roth. Registering images to untextured geometry using average shading gradients. In IEEE Conference on Computer Vision and Pattern Recognition (CVPR), pages 2030-2038, 2015.

25. V. Powers and T. Wörmann. An algorithm for sums of squares of real polynomials. Journal of Pure and Applied Algebra, 127(1):99-104, 1998.

26. Mihai Putinar. Positive polynomials on compact semialgebraic sets. In Indiana Univ. Math. J., pages 969-984, 1993.

27. Szymon Rusinkiewicz and Marc Levoy. Efficient variants of the ICP algorithm. In 3-D Digital Imaging and Modeling (3DIM), pages 145-152, 2001.

28. Grant Schindler, Panchapagesan Krishnamurthy, Roberto Lublinerman, Yanxi Liu, and Frank Dellaert.
Detecting and matching repeated patterns for automatic geo-tagging in urban environments. In IEEE Conference on Computer Vision and Pattern Recognition (CVPR), pages $1-7,2008$.

29. Gerald Schweighofer and Axel Pinz. Globally optimal $\mathrm{o}(\mathrm{n})$ solution to the pnp problem for general camera models. In The British Machine Vision Conference (BMVC), pages 1-10, 2008.

30. Aleksandr V. Segal, Dirk Haehnel, and Sebastian Thrun. Generalized-icp. In Robotics: Science and Systems (RSS), 2009.

31. M. Tamaazousti, V. Gay-Bellile, S. N. Collette, S. Bourgeois, and M. Dhome. Nonlinear refinement of structure from motion reconstruction by taking advantage of a partial knowledge of the environment. In IEEE Conference on Computer Vision and Pattern Recognition (CVPR), pages 3073-3080, 2011.

32. Aparna Taneja, Luca Ballan, and Marc Pollefeys. Cityscale change detection in cadastral $3 \mathrm{~d}$ models using images. In IEEE Conference on Computer Vision and Pattern Recognition (CVPR), pages 113-120, 2013.

33. Adrien Bartoli Umberto Castellani. 3d shape registration. In $3 D$ Imaging, Analysis, and Applications, Springer, pages 221-264, 2012.

34. Jan Verschelde. Algorithm 795: Phcpack: A generalpurpose solver for polynomial systems by homotopy continuation. ACM Transactions on Mathematical Software (TOMS), 25(2):251-276, 1999.

35. S. Wagner. Archimedean quadratic modules: A decision problem for real multivariate polynomials. Ph.D. thesis, Universität Konstanz, August 2009.

36. Jiaolong Yang, Hongdong Li, and Yunde Jia. Go-icp: Solving 3d registration efficiently and globally optimally. In IEEE International Conference on Computer Vision (ICCV), pages 1457-1464, December 2013.

37. Jiaolong Yang, Hongdong Li, and Yunde Jia. Optimal essential matrix estimation via inlier-set maximization. In European Conference on Computer Vision (ECCV), pages 111-126, 2014.

38. Xi Zhang, G. Agam, and Xin Chen. Alignment of 3d building models with satellite images using extended chamfer matching. In IEEE Conference on Computer Vision and Pattern Recognition Workshops (CVPRW), pages 746-753, 2014. 